\title{
Polynomial approach to explicit formulae for generalized binomial coefficients
}

\author{
Fedor Petrov ${ }^{1,2}$ (D)
}

Received: 27 October 2015 / Revised: 20 January 2016 / Accepted: 2 February 2016 /

Published online: 2 March 2016

(C) Springer International Publishing AG 2016

\begin{abstract}
We extend the polynomial approach to the hook length formula proposed in Károlyi et al. Adv Math 277:252-282 (2015) to several other problems of the same type, including the number of paths formula in the Young graph of strict partitions.
\end{abstract}

Keywords Combinatorial Nullstellensatz $\cdot$ Polynomial identities $\cdot$ Multidimensional interpolation · Generalized binomial coefficients · Graded graph

Mathematics Subject Classification $\quad 05 \mathrm{E} 05 \cdot 05 \mathrm{E} 40 \cdot 20 \mathrm{C} 30$

The multivariate polynomial interpolation, or, in other words, the explicit form of Alon's Combinatorial Nullstellensatz $[9,13]$, recently proved to be powerful in proving polynomial identities: in [9] it was used for the direct short proof of Dyson's conjecture, later generalized in [10] for the equally short proof of the $q$-version of Dyson's conjecture, then after additional combinatorial work it allowed to prove identities of Morris, Aomoto, Forrester (the last was open), their common generalizations, both in classical and $q$-versions.

Here we outline how this method works in classical theory of symmetric functions, "self-proving" polynomial identities corresponding to the counting paths in the Young graph and "strict Young graph", or, in other words, counting dimensions of linear and projective representations of symmetric groups.

Supported by the Russian Scientific Foundation grant 14-11-00581.

Fedor Petrov

fedyapetrov@gmail.com

1 St. Petersburg Department of V.A. Steklov Institute of Mathematics of the Russian Academy of Sciences, 27 Fontanka nab., St. Petersburg 191023, Russia

2 Saint Petersburg State University, 7/9 Universitetskaya nab., St. Petersburg 199034, Russia 
The interpolation-based approach to symmetric functions has been earlier developed by Olshanski, Borodin, Okounkov, Vershik, Regev [5, 14-16] and others. It may sound speculatively, but according to author's opinion, the novelty of my/author's approach consists in considering arbitrary polynomials (rather antisymmetric, than symmetric) as functions of usual points and applying general facts about general (not symmetric) polynomials instead of considering "symmetric functions as functions of partitions" (formulation from [5]).

We start with a general framework of paths in graded graphs.

\section{Graded graphs}

Let $G$ be a $\mathbb{Z}$-graded countable directed graph with the vertex set $V(G)=\bigsqcup_{i=-\infty}^{\infty} V_{i}$, directed edges $(u, v) \in E(G)$ joining vertices of the consecutive levels $u \in V_{i}$ and $v \in V_{i+1}$ for some integer $i$. In what follows both indegrees and outdegrees of all vertices are bounded. This implies that the number $P_{G}(v, u)$ of directed paths from $v$ to $u$ is finite for any two fixed vertices $v, u$. This number is known as the generalized binomial coefficient (usual binomial coefficient appears for the Pascal triangle).

Fix a positive integer $k$. Our examples are induced subgraphs of the lattice $\mathbb{Z}^{k}$. Vertices are graded by the sum of coordinates, edges correspond to increasing of any coordinate by 1 . That is, indegree of any vertex equals its outdegree and equals $k$. It is convenient to identify the vertex set $V$ with the monomials $x_{1}^{c_{1}} \cdots x_{k}^{c_{k}},\left(c_{1}, \ldots, c_{k}\right) \in$ $\mathbb{Z}^{k}$. Then any edge corresponds to multiplying of monomial by some variable $x_{i}$. Finite linear combinations of elements of $V$ (with, say, rational coefficients, it is not essential hereafter) form a ring $\mathbb{Q}\left[x_{1}, x_{1}^{-1}, \ldots, x_{k}, x_{k}^{-1}\right]$ of Laurent polynomials in variables $x_{1}, \ldots, x_{k}$. Not necessary finite linear combinations form a $\mathbb{Q}\left[x_{1}, x_{1}^{-1}, \ldots, x_{k}, x_{k}^{-1}\right]$ module which we denote by $\Phi$. For monomial $v \in V$ and $\varphi \in \Phi$ we denote by $[v] \varphi$ the coefficient of $v$ in the series $\varphi$.

Define the minimum of two monomials $u=\prod x_{i}^{a_{i}}$ and $v=\prod x_{i}^{b_{i}}$ as $\min (u, w)=$ $\prod x_{i}^{\min \left(a_{i}, b_{i}\right)}$. If $\min (u, v)=u$ we say that the monomial $v$ majorates $u$.

Now we describe three examples of graded graphs for which explicit formulae for generalized binomial coefficients are known.

- Multidimensional Pascal graph $\mathcal{P}_{k}$. This is a subgraph of $\mathbb{Z}^{k}$ formed by the vectors with integral non-negative coordinates (or, in the monomial language, by monomials having all variables in non-negative power). For $k=2$ this graph is isomorphic to the Pascal triangle.

- Restricted Young graph $y_{k}$. This is a subgraph of the Pascal graph formed by vectors $\left(c_{1}, \ldots, c_{k}\right)$ with strictly increasing non-negative coordinates, $0 \leqslant c_{1}<c_{2}<\cdots$ $<c_{k}$. The vertices of $y_{k}$ may be identified with the Young diagrams having at most $k$ rows (to any vertex $\left(c_{1}, \ldots, c_{k}\right)$ of $y_{k}$ we assign a Young diagram with rows (to any vertex $\left(c_{1}, \ldots, c_{k}\right)$ of $y_{k}$ we assign a Young diagram with lengths of rows $\left.c_{1} \leqslant c_{2}-1 \leqslant \cdots \leqslant c_{k}-(k-1)\right)$. Edges correspond to addition of boxes. The usual Young graph has all Young diagrams as vertices, but when we count number of paths between two diagrams we may always restrict ourselves to $y_{k}$ with large enough $k$. 
- Graph of strict partitions $\delta y_{k}$. This is a subgraph of the Pascal graph formed by vectors $\left(c_{1}, \ldots, c_{k}\right)$ with non-strictly increasing non-negative coordinates $0 \leqslant$ $c_{1} \leqslant c_{2} \leqslant \cdots \leqslant c_{k}$ satisfying the following condition: if $c_{i}=c_{j}$ for $i \neq j$, then $c_{i}=0$. To any vertex $\left(c_{1}, \ldots, c_{k}\right)$ we may assign a Young diagram with lengths of rows $c_{1}, \ldots, c_{k}$. So, this diagram has at most $k$ (non-empty) rows and they have distinct lengths.

The following general straightforward fact connects generalized binomial coefficients for subgraphs of $\mathbb{Z}^{k}$ with coefficients of polynomials or power series.

Theorem 1.1 Let $G$ be a subgraph of $\mathbb{Z}^{k}$, monomials $u, v \in V(G)$ be two vertices of $G, \operatorname{deg} u \geqslant \operatorname{deg} v$. Assume that $\varphi \in \Phi$ is a series satisfying the following conditions:

(i) $[v] \varphi=1$;

(ii) if $v^{\prime} \in V(G)$ and $\operatorname{deg} v=\operatorname{deg} v^{\prime}$, then $\left[v^{\prime}\right] \varphi=0$;

(iii) if $w \notin V(G)$, but $x_{i} w \in V(G)$ for some $x_{i}$, then $[w] \varphi \cdot\left(x_{1}+\cdots+x_{k}\right)^{\operatorname{deg} w-\operatorname{deg} v}$ $=0$.

Then the number of paths from $v$ to $u$ equals

$$
P_{G}(v, u)=[u] \varphi \cdot\left(x_{1}+\cdots+x_{k}\right)^{\operatorname{deg} u-\operatorname{deg} v} .
$$

Proof Induction on $\operatorname{deg} u$. The base $\operatorname{deg} u=\operatorname{deg} v$ follows from (i) and (ii). Denote $m=\operatorname{deg} u-\operatorname{deg} v$ and assume that the statement is proved for all vertices of degree less than $m+\operatorname{deg} v$. Let $\left(u_{i}, u\right), i=1, \ldots, s$, be all edges of the graph $G$ coming to $u$. Clearly $s \leqslant k$ and without loss of generality $u=x_{i} u_{i}$ for $i=1, \ldots, s$. Also note that for $s<i \leqslant k$ we have $\left[u x_{i}^{-1}\right] \varphi \cdot\left(x_{1}+\cdots+x_{k}\right)^{m-1}=0$ by property (iii). Thus

$$
\begin{aligned}
{[u] \varphi \cdot\left(x_{1}+\cdots+x_{k}\right)^{m} } & =\sum_{i=1}^{k}\left[u x_{i}^{-1}\right] \varphi \cdot\left(x_{1}+\cdots+x_{k}\right)^{m-1} \\
& =\sum_{i=1}^{s}\left[u_{i}\right] \varphi \cdot\left(x_{1}+\cdots+x_{k}\right)^{m-1} \\
& =\sum_{i=1}^{s} P_{G}\left(v, u_{i}\right)=P_{G}(v, u)
\end{aligned}
$$

as desired.

Remark 1.2 In other words, both parts of (1) are fundamental solutions of the Laplace equation on the part of our graph starting from the level of $v$.

Theorem 1.1 leads to a natural question: for which labeled graphs $(G, u)$ there exists a function $\varphi$ such that conditions (i)-(iii) are satisfied? We do not know a full answer. The following statement is at least general enough to cover all examples of this paper.

Theorem 1.3 Assume that $V \subset \mathbb{Z}^{k}, G$ is the induced subgraph of $\mathcal{P}_{k}$ with vertex set $V=V(G)$ and it satisfies the following two conditions: 
(i) (minimum-closed set) if $u, w \in V$, then also $\min (u, w) \in V$.

(ii) (coordinate convexity) if $u, w \in V, w=u x_{i}^{m}$ for some index $i$ and positive integer $m$, then also $u x_{i}^{s} \in V$ for all $0 \leqslant s \leqslant m$.

Then for any $v \in V$ there exists a function $\varphi$ satisfying conditions of Theorem 1.1.

Proof Call a monomial $u \in \mathbb{Z}^{k}$ special if either $u \in V, \operatorname{deg} u=\operatorname{deg} v$ (in particular, $v$ itself is special), or $\operatorname{deg} u \geqslant \operatorname{deg} v, u \notin V, x_{i} u \in V$ for some variable $x_{i}$. It suffices to prove that there exists a function $\varphi$ homogeneous of degree deg $v$ with any prescribed values of $[u]\left(\sum x_{i}\right)^{\operatorname{deg} u-\operatorname{deg} v} \varphi$ for all special $u$. This is a linear system on coefficients of $\varphi$.

By replacing $V$ with $v^{-1} V$ we may suppose that $v=1$. For any special monomial $u$ define its son $S(u)$ as follows: if $\operatorname{deg} u=0$, then $S(u)=0$, if $u \notin V, x_{i} u \in V$, then $S(u)=x_{i}^{-\operatorname{deg} u} u$ (if different indexes $i$ satisfy $x_{i} u \in V$, choose any).

If $u \neq w$ are two special monomials and $w$ majorates $S(u)$, then $\operatorname{deg} w>\operatorname{deg} u$. Indeed, assume that on the contrary $\operatorname{deg} w \leqslant \operatorname{deg} u$. First, if $\operatorname{deg} u=0$, then $\operatorname{deg} w=0$ and $w=S(u)=u$, a contradiction. Thus $d=\operatorname{deg} u>0$, we may suppose that $x_{1} u \in V, S(u)=x_{1}^{-d} u$. Denote $w=x_{1}^{a_{1}-d} x_{2}^{a_{2}} \cdots x_{k}^{a_{k}} u, a_{i} \geqslant 0, d \geqslant a_{1}+\cdots+a_{k}$, and either $w \in V$ or $x_{i} w \in V$ for some $i$. Since $w \neq u$, we have $d>a_{1}$. Denote $u_{0}=$ $\min \left(x_{i} w, x_{1} u\right)$ if $x_{i} w \in V$, and $u_{0}=\min \left(w, x_{1} u\right)$ if $w \in V$. Then $u_{0}=x_{1}^{a_{1}-d+\varepsilon} u$, where $\varepsilon \in\{0,1\}$, and $u_{0} \in V$ since $V$ is a minimum-closed set. The coordinate convexity implies that if both $u_{0}, x_{1} u$ belong to $V$, so does $u$. A contradiction.

Now we start to solve our linear system for coefficients of $\varphi$. For any special monomial $u$ we have a linear relation on the monomials of degree 0 majorated by $u$. Between them there is a monomial $S(u)$, and it does not appear in relations corresponding to special monomials $w \neq u$ with $\operatorname{deg} w \leqslant \operatorname{deg} u$. It allows to fix coefficients of $\varphi$ in the appropriate order (by increasing the degree of $u$ ) and fulfil all our relations.

\section{Observation on polynomials}

Recall the Combinatorial Nullstellensatz of Alon [1].

Theorem 2.1 (Combinatorial Nullstellensatz) Let $K$ be a field and $A_{i}, i=1, \ldots, k$, be non-empty subsets of $K,\left|A_{i}\right|=d_{i}+1$. Let $f\left(x_{1}, \ldots, x_{k}\right) \in K\left[x_{1}, \ldots, x_{k}\right]$ be a polynomial of degree at most $d_{1}+\cdots+d_{k}$ such that $f\left(c_{1}, \ldots, c_{k}\right)=0$ for all points $\left(c_{1}, \ldots, c_{k}\right) \in A_{1} \times A_{2} \times \cdots \times A_{k}$. Then

$$
\left[x_{1}^{d_{1}} x_{2}^{d_{2}} \cdots x_{k}^{d_{k}}\right] f\left(x_{1}, \ldots, x_{k}\right)=0 .
$$

For verifying polynomial identities which allow to calculate coefficients we use the following observation in the spirit of the Combinatorial Nullstellensatz.

Let $K$ be a field and $A_{i}=\left\{a_{i 0}, a_{i 1}, \ldots, a_{i n}\right\} \subset K, i=1, \ldots, k$, be its subsets of size $\left|A_{i}\right|=n+1$.

Observation 2.2 A polynomial $f\left(x_{1}, \ldots, x_{k}\right) \in K\left[x_{1}, \ldots, x_{k}\right]$ of degree at most $n$ is uniquely determined by its values on the combinatorial simplex

$$
\Delta=\left\{A\left(t_{1}, \ldots, t_{k}\right)=\left(a_{1 t_{1}}, a_{2 t_{2}}, \ldots, a_{k t_{k}}\right), \sum t_{i} \leqslant n\right\} .
$$


Proof The number of points in $\Delta$ equals exactly the dimension of the space of polynomials with degree at most $n$ in $k$ variables. Hence it suffices to check either existence or uniqueness of the polynomial with degree at most $n$ and with prescribed values on $\Delta$. Both tasks are easy as we may see:

Existence. Induction on $n$. The base $n=0$ is clear. Assume that $n>1$ and for the simplex $\Delta^{\prime}$, which corresponds to the inequality $\sum t_{i} \leqslant n-1$, there exists a polynomial $f\left(x_{1}, \ldots, x_{k}\right)$ with degree at most $n-1$ and with prescribed values on $\Delta^{\prime}$. For any point $A\left(t_{1}, \ldots, t_{k}\right)$ with $\sum t_{i}=n$ we have a polynomial

$$
\prod_{\substack{1 \leqslant i \leqslant k \\ 0 \leqslant s_{i}<t_{i}}}\left(x_{i}-a_{i s_{i}}\right)
$$

vanishing on all points of $\Delta$ but $A\left(t_{1}, \ldots, t_{k}\right)$. The appropriate linear combination of $f(x)$ and such polynomials gives a polynomial with the prescribed values on $\Delta$ (and degree at most $n$ ).

Uniqueness. It suffices to prove that the polynomial $f\left(x_{1}, \ldots, x_{k}\right)$ with degree at most $n$, which vanishes on $\Delta$, identically equals 0 . Assume the contrary. Let $x_{1}^{t_{1}} \cdots x_{k}^{t_{k}}$ be a highest degree term in $f$. The set $\Delta$ contains the product $B_{1} \times \cdots \times B_{k}$, where $B_{i}=\left\{a_{i 0}, \ldots, a_{i t_{i}}\right\},\left|B_{i}\right|=t_{i}+1$. By the Combinatorial Nullstellensatz, $f$ cannot vanish on $\prod B_{i}$. A contradiction.

For $A_{i}=\{0,1, \ldots, n\}$ we get the standard simplex

$$
\Delta_{k}^{n}=\left\{\left(t_{1}, \ldots, t_{k}\right): t_{i} \geqslant 0, t_{i} \in \mathbb{Z}, \sum t_{i} \leqslant n\right\}
$$

It is the main partial case for us. In the theory of $q$-identities its $q$-analogue, which corresponds to the set $\left\{1, q, \ldots, q^{n}\right\}$, plays an analogous role.

We use notations $x^{\underline{n}}=x(x-1) \cdots(x-n+1)$ and $\left(\begin{array}{l}x \\ n\end{array}\right)=x \underline{n} / n !$.

Remark 2.3 Some other notations, including $x^{\downarrow n},\left(x\lfloor n),(x \mid n),(x)_{n}\right.$ are used for falling factorials. I/author prefer the Capelli-Toscano notation, popularized by Knuth, see his arguments in [12]. The author finds it quite intuitive, particularly in the context of this paper.

The following particular case of interpolation on $\Delta_{k}^{n}$ appears to be useful.

Lemma 2.4 If $f \in K\left[x_{1}, \ldots, x_{k}\right]$, deg $f \leqslant n$ and $f$ vanishes on $\Delta_{k}^{n-1}$, then

$$
\begin{aligned}
f\left(x_{1}, \ldots, x_{k}\right) & =\sum_{c_{1}+\cdots+c_{k}=n} f\left(c_{1}, \ldots, c_{k}\right) \cdot \prod_{i=1}^{k}\left(\begin{array}{l}
x_{i} \\
c_{i}
\end{array}\right) \\
& =\sum_{c_{1}+\cdots+c_{k}=n} \frac{f\left(c_{1}, \ldots, c_{k}\right)}{c_{1} ! \cdots c_{k} !} \cdot \prod_{i=1}^{k} x_{i} c_{i} .
\end{aligned}
$$


Proof It suffices to check the equality for values on $\Delta_{k}^{n}$. Both parts vanish on $\Delta_{k}^{n-1}$. If $\left(x_{1}, \ldots, x_{k}\right)$ is a point on $\Delta_{k}^{n} \backslash \Delta_{k}^{n-1}$, i.e. $\sum x_{i}=n$, then all summands on the right vanish except (possibly) the summand with $c_{i}=x_{i}$ for all $i$, and its value just equals $f\left(c_{1}, \ldots, c_{k}\right)=f\left(x_{1}, \ldots, x_{k}\right)$, i.e. the value of LHS at the same point, as desired.

We start our series of applications of Observation 2.2 along with the multinomial version of the $\mathrm{Chu}-$ Vandermonde identity.

\subsection{Example: Chu-Vandermonde identity and multinomial coefficient}

$$
\left(\begin{array}{c}
x_{1}+\cdots+x_{k} \\
n
\end{array}\right)=\sum_{c_{1}+\cdots+c_{k}=n} \prod_{i=1}^{k}\left(\begin{array}{l}
x_{i} \\
c_{i}
\end{array}\right) .
$$

This immediately follows from Lemma 2.4 .

Identity (2) has the following form in falling factorials:

$$
\left(x_{1}+\cdots+x_{k}\right)^{n}=\sum_{m_{1}+\cdots+m_{k}=n} \frac{n !}{\prod m_{i} !} \prod_{i=1}^{k} x_{i} \frac{m_{i}}{} .
$$

Taking only the leading terms in both sides we get the Multinomial Theorem, i.e.

$$
\left(x_{1}+\cdots+x_{k}\right)^{n}=\sum_{m_{1}+\cdots+m_{k}=n} \frac{n !}{\prod m_{i} !} \prod_{i=1}^{k} x_{i}^{m_{i}} .
$$

Returning back to graded graphs, for the multidimensional Pascal graph $\mathcal{P}_{k}$ we get the following formula for the number of paths from the origin 1 to any vertex $v=\prod x_{i}^{m_{i}}$, $m_{i} \geqslant 0, n=\sum m_{i}=\operatorname{deg} v$ :

$$
P_{\mathcal{P}_{k}}(1, v)=[v]\left(\sum x_{i}\right)^{n}=\frac{n !}{\prod m_{i} !} .
$$

This immediately follows from Theorem 1.1 for $\varphi=1$ and identity (4).

\section{Hook length formula}

We start with a polynomial identity which is in a sense similar to the $\mathrm{Chu}$-Vandermonde identity (3).

\section{Theorem 3.1}

$$
\begin{aligned}
\prod_{i<j}\left(x_{j}-x_{i}\right)\left(\sum_{i=1}^{k} x_{i}-\frac{k(k-1)}{2}\right)^{\underline{n}} & \\
& =\sum_{n_{1}+\cdots+n_{k}=n+k(k-1) / 2} \frac{n !}{\prod n_{i} !} \prod_{i<j}\left(n_{j}-n_{i}\right) \cdot \prod x_{i} \frac{n_{i}}{}
\end{aligned}
$$


Proof By Lemma 2.4, it suffices to check that LHS vanishes on $\Delta_{k}^{n+k(k-1) / 2-1}$. Let $x_{i}$ be non-negative integers and $\sum x_{i}<n+k(k-1) / 2$. If $\sum x_{i}<k(k-1) / 2$ then some factor $x_{i}-x_{j}$ vanishes, otherwise $y=\sum x_{i}-k(k-1) / 2$ is non-negative and $y<n$, hence $y^{\underline{n}}=0$.

As in the Chu-Vandermonde case, we may take only the leading terms and get the identity

$$
\begin{aligned}
\prod_{i<j}\left(x_{j}-x_{i}\right) & \left(x_{1}+\cdots+x_{k}\right)^{n} \\
& =\sum_{n_{1}+\cdots+n_{k}=n+k(k-1) / 2} \frac{n !}{\prod n_{i} !} \prod_{i<j}\left(n_{j}-n_{i}\right) \cdot \prod x_{i}{ }^{n_{i}} .
\end{aligned}
$$

Being divided by $\prod_{i<j}\left(x_{j}-x_{i}\right)$, this becomes a well-known expansion

$$
\left(x_{1}+x_{2}+\cdots\right)^{n}=\sum_{\lambda \vdash n} \operatorname{dim} \lambda \cdot s_{\lambda}
$$

for the Schur symmetric functions (combined with the Frobenius formula for dimensions).

Corollary 3.2 (Frobenius dimension formula) For any vertex $v=x_{1}^{n_{1}} \cdots x_{k}^{n_{k}}, \sum n_{i}=$ $n+k(k-1) / 2$, of the Young graph $y_{k}$ the number $P_{y_{k}}\left(v_{0}, v\right)$ of paths to this vertex from $v_{0}=(0,1, \ldots, k-1)$ equals

$$
P y_{k}\left(v_{0}, v\right)=\frac{n !}{\prod n_{i} !} \prod_{i<j}\left(n_{j}-n_{i}\right)=[v] \prod_{i<j}\left(x_{j}-x_{i}\right)\left(\sum x_{i}\right)^{n}
$$

Proof Take $\varphi=\prod_{i<j}\left(x_{j}-x_{i}\right)$ and apply Theorem 1.1. Conditions (i) and (ii) follow from (5) with $n=0$ (actually, this is just the Vandermonde determinant formula). For checking (iii), note that such $w=\prod x_{i}^{n_{i}}$ satisfies either $n_{i}<0$ for some $i$ or $n_{i}=n_{j}$ for some $i, j$. In both cases (5) yields that the corresponding coefficient vanishes.

Note that our proof of (6) does not use the Multinomial Theorem, but is proved in the same way and the proof is almost equally short. Identity (5) appears also in the important for the development of the polynomial method paper [2], where it is used for appropriate application of the Combinatorial Nullstellensatz, while we show how it may be proved by the (explicit version of) Combinatorial Nullstellensatz.

In the rest part of this section we explain the relation of (6) with hook lengths of the Young diagram, this is mostly for the sake of completeness.

Recall that the graph $y_{k}$ may be viewed as the graph of Young diagrams having at most $k$ rows. For a vertex $v \in y_{k}, v=x_{1}^{n_{1}} \cdots x_{k}^{n_{k}}$, the corresponding diagram $\lambda(v)$ has $k$ (possibly empty) rows with lengths $n_{1} \leqslant n_{2}-1 \leqslant \cdots \leqslant n_{k}-(k-1)$. Edges of the graph $y_{k}$ correspond to adding boxes, and paths correspond to the skew standard Young tableaux: for any path with, say $m$ edges, put numbers $1,2, \ldots, m$ in the corresponding adding boxes. In this language, expression (6) counts the number of 
standard Young tableaux of the shape $\lambda(v)$. Assuming $n_{1}>0$ (i.e. the number of rows equals $k$ ), we may interpret parameters $n_{1}, \ldots, n_{k}$ as hook lengths of $k$ boxes in the first column. Recall that (now specify that the largest column in the Young diagram is the leftmost and the largest row is the lowest) a hook of a box $X$ in the Young diagram is a union of $X$; all boxes in the same column which are higher than $X$; all boxes in the same row which are on the right to $X$.

Claim In the above notations the product of hook lengths of all boxes in the Young diagram $\lambda(v)$ equals

$$
\prod h(\square)=\frac{\prod n_{i} !}{\prod_{i<j}\left(n_{j}-n_{i}\right)}
$$

Proof Assume that boxes $a, b$ of the Young diagram lie in the same row and $a, c$ in the same column. Let $d$ be a box such that $a b d c$ is a rectangle. If $d$ belongs to the diagram then $h(a)<h(b)+h(c)$, otherwise $h(a)>h(b)+h(c)$. Hence we always have the inequality $h(a) \neq h(b)+h(c)$. Now $n_{1}, \ldots, n_{k}$ are hook lengths of boxes in the first column. In the $i$-th row there are $n_{i}-(i-1)$ boxes, and their hook lengths are distinct numbers from 1 to $n_{i}$, with $i-1$ values excluded, and those excluded values are $n_{i}-n_{1}, n_{i}-n_{2}, \ldots, n_{i}-n_{i-1}$, by the inequality. It remains to multiply over $i=1,2, \ldots, k$.

The above claim allows to formulate Corollary 3.2 in the form of the hook length formula [6].

Theorem 3.3 (hook length formula) The number of the standard Young tableaux of a given shape $\lambda$ with $n$ boxes equals $n ! / \prod \square h(\square)$, where the product is taken over all boxes of $\lambda$.

Remark 3.4 As the referee pointed out, Theorem 3.1 may be derived from the formalism of [5] combined with the Frobenius dimension formula.

\section{Skew Young tableaux}

Here we get generalizations of Theorem 3.1, corresponding to counting paths between two arbitrary vertices of $y_{k}$ (i.e. the number of skew Young tableaux of a given shape).

The role of the Vandermonde determinant $\prod_{i<j}\left(x_{j}-x_{i}\right)=\operatorname{det}\left(x_{i}^{j-1}\right)_{i, j}$ is played by alternating determinants

$$
a_{m_{1}, \ldots, m_{k}}\left(x_{1}, \ldots, x_{k}\right)=\operatorname{det}\left(x_{i}^{m_{j}}\right), \quad b_{m_{1}, \ldots, m_{k}}\left(x_{1}, \ldots, x_{k}\right)=\operatorname{det}\left(x_{i} \frac{m_{j}}{}\right) .
$$

The following identity specializes to Theorem 3.1 for $m_{i}=i-1, i=1,2, \ldots, k$. 
Theorem 4.1 If $m_{1}<\cdots<m_{k}$ are distinct non-negative integers, $m=\sum m_{i}$, then

$$
\begin{aligned}
b_{m_{1}, \ldots, m_{k}}\left(x_{1}, \ldots, x_{k}\right) & \left(\sum x_{i}-m\right)^{\underline{n}} \\
= & \sum_{n_{1}+\cdots+n_{k}=n+m} \frac{n !}{\prod n_{i} !} b_{m_{1}, \ldots, m_{k}}\left(n_{1}, \ldots, n_{k}\right) \cdot \prod x_{i} \frac{n_{i}}{} .
\end{aligned}
$$

Proof Due to Lemma 2.4, it suffices to check that LHS vanishes on $\Delta_{k}^{n+m-1}$. Fix a point $\left(x_{1}, \ldots, x_{k}\right) \in \Delta_{k}^{n-1}$. Let $y_{1} \leqslant \cdots \leqslant y_{k}$ be the increasing permutation of $x_{i}^{\prime}$. If $y_{i}<m_{i}$ for some $i$, then the matrix $\left(y_{i} \frac{m_{j}}{-}\right)$ is singular as it has $i \times(n-i+1)$ minor of zeros, and the matrix $\left(x_{i} \frac{m_{j}}{2}\right)$ is therefore singular too. If $y_{i} \geqslant m_{i}$ for all $i$, then denoting $y=\sum x_{i}-m=\sum\left(y_{i}-m_{i}\right) \geqslant 0$ we have $0 \leqslant y<n$, hence $y^{\underline{n}}=0$.

Taking the leading terms we get the following identity for homogeneous polynomials:

$$
\begin{aligned}
a_{m_{1}, \ldots, m_{k}}\left(x_{1}, \ldots, x_{k}\right) & \left(\sum x_{i}\right)^{n} \\
= & \sum_{n_{1}+\cdots+n_{k}=n+m} \frac{n !}{\prod n_{i} !} b_{m_{1}, \ldots, m_{k}}\left(n_{1}, \ldots, n_{k}\right) \cdot \prod x_{i}{ }^{n_{i}} .
\end{aligned}
$$

Corollary 4.2 (skew dimension formula) Let $v_{1}=x_{1}^{m_{1}} \cdots x_{k}^{m_{k}}, v_{2}=x_{1}^{n_{1}} \cdots x_{k}^{n_{k}}$ be two vertices of the Young graph $y_{k}$ such that $n_{i} \geqslant m_{i}$ for all $i=1,2, \ldots, k$. Denote $\sum m_{i}=m, \sum n_{i}=n+m$. Then the number $P y_{k}\left(v_{1}, v_{2}\right)$ of paths from $v_{1}$ to $v_{2}$ equals

$$
\begin{aligned}
P y_{k}\left(v_{1}, v_{2}\right) & =\frac{n !}{\prod n_{i} !} b_{m_{1}, \ldots, m_{k}}\left(n_{1}, \ldots, n_{k}\right) \\
& =\left[v_{2}\right] a_{m_{1}, \ldots, m_{k}}\left(x_{1}, \ldots, x_{k}\right)\left(\sum x_{i}\right)^{n} .
\end{aligned}
$$

Proof Take $\varphi=a_{m_{1}, \ldots, m_{k}}\left(x_{1}, \ldots, x_{k}\right)$ and apply Theorem 1.1. Conditions (i) and (ii) follow from (7) with $n=0$ (or from expanding the determinant). For checking (iii), note that such $w=\prod x_{i}^{n_{i}}$ satisfies either $n_{i}<0$ for some $i$ or $n_{i}=n_{j}$ for some $i, j$. In both cases (7) yields that the corresponding coefficient vanishes.

Remark 4.3 This formula for the number of paths between two arbitrary vertices of the Young graph appeared in [15, Theorem 8.1], see also [16]. Recently it appeared in the context of additive combinatorics in [3, Lemma 4], [4].

The value $b_{m_{1}, \ldots, m_{k}}\left(n_{1}, \ldots, n_{k}\right)$ has a combinatorial interpretation following from the Lindström-Gessel-Viennot Lemma: up to a multiple $\prod m_{i}$ ! it is a number of semistandard Young tableaux of a given shape and content. See details in [7].

A.M. Vershik pointed out that similar results are known for the graph of strict diagrams. It also may be included in our framework. 


\section{Strict diagrams}

For counting the number of paths in the graph $\delta y_{k}$ we need series which are not polynomials.

Let $x_{1}, \ldots, x_{k}$ be variables (as before). Consider the set $\mathcal{M}$ of rational functions in those variables with denominator $\prod_{i<j}\left(x_{i}+x_{j}\right)$. Expand such functions in Laurent series in $x_{1}, x_{2} / x_{1}, x_{3} / x_{2}, \ldots, x_{k} / x_{k-1}$ (i.e., $\left(x_{i}+x_{j}\right)^{-1}=x_{i}^{-1}-x_{j} x_{i}^{-2}+x_{j}^{2} x_{i}^{-3}-$ $\cdots$ for $i<j)$. Define the value of such function at a point $\left(c_{1}, \ldots, c_{k}\right)$ with nonnegative coordinates as follows: if coordinates are positive, just substitute them in the function, if some coordinates vanish, replace them by positive numbers $t, t^{2}, \ldots$ (in such order), and let $t$ tend to +0 . What we actually need is that for $i<j$ the value $x_{j} /\left(x_{i}+x_{j}\right)$ with $x_{i}=x_{j}=0$ equals 0 . Each function $f \in \mathcal{M}$ may be expanded as $f=P[f]+Q[f]$, where $P[f]$ is a polynomial in $x_{1}, \ldots, x_{k}$, and in $Q[f]$ each term $\prod x_{i}^{c_{i}}$ contains at least one variable $x_{i}$ in a negative power $c_{i}<0$. We say that $P[f]$ is the polynomial component of $f$ and $Q[f]$ is the antipolynomial component of the function $f\left(x_{1}, \ldots, x_{k}\right)$.

Lemma 5.1 Define a function $f_{n}\left(x_{1}, \ldots, x_{k}\right) \in \mathcal{M}$ by the formula

$$
f_{n}\left(x_{1}, \ldots, x_{k}\right)=\prod_{1 \leqslant i<j \leqslant k} \frac{x_{i}-x_{j}}{x_{i}+x_{j}} \cdot\left(x_{1}+\cdots+x_{k}\right)^{n} .
$$

Then

(i) its antipolynomial component $Q\left[f_{n}\right]$ vanishes on the standard simplex $\Delta_{k}^{n}$,

(ii) if $c_{1}, \ldots, c_{k}$ are integers such that $c_{j}<0, c_{i} \geqslant 0$ for $i=j+1, \ldots, k$, then $\left[\prod x_{i}^{c_{i}}\right] f_{n}=0$.

Proof We may suppose that $k=2 d$ is even (else replace $k$ with $k+1$ and put $x_{k+1}=0$ ). Consider the following antisymmetric $k \times k$ matrix: $a_{i j}=\left(x_{i}-x_{j}\right) /\left(x_{i}+x_{j}\right)$. Note that its Pfaffian lies in $\mathcal{M}$, it is homogeneous of order 0 , it vanishes for $x_{i}=x_{j}$ and is singular for $x_{i}=-x_{j}$. Thus up to a constant multiple (it is not hard to verify that actually up to a sign) it equals $\prod_{1 \leqslant i<j \leqslant k}\left(x_{i}-x_{j}\right) /\left(x_{i}+x_{j}\right)$. The Pfaffian is an alternating sum of expressions like

$$
\prod_{i=1}^{d} \frac{\xi_{i}-\zeta_{i}}{\xi_{i}+\zeta_{i}}
$$

where $\left\{\xi_{1}, \zeta_{1}, \ldots, \xi_{d}, \zeta_{d}\right\}=\left\{x_{1}, \ldots, x_{k}\right\}$. On the other hand, the Vandermonde identity (2) allows to express the falling factorial $\left(x_{1}+\cdots+x_{k}\right)^{n}$ as a linear combination of expressions like

$$
\prod_{i=1}^{d}\left(\xi_{i}+\zeta_{i}\right) \frac{\alpha_{i}}{,}, \quad \alpha_{i} \geqslant 0, \quad \alpha_{1}+\cdots+\alpha_{d}=n
$$


Fixing at first the partition into pairs and next the exponents $\alpha_{1}, \ldots, \alpha_{d}$, we reduce both claims to the expression

$$
F\left(x_{1}, \ldots, x_{k}\right)=\prod_{i=1}^{d} \frac{\xi_{i}-\zeta_{i}}{\xi_{i}+\zeta_{i}} \cdot\left(\xi_{i}+\zeta_{i}\right) \frac{\alpha_{i}}{.}
$$

Note that variables are separated here, thus the polynomial component of this product is just the product of polynomial components of the factors. We have

$$
Q[F]=F-P[F]=\prod_{i=1}^{d} \frac{\xi_{i}-\zeta_{i}}{\xi_{i}+\zeta_{i}} \cdot\left(\xi_{i}+\zeta_{i}\right) \frac{\alpha_{i}}{-}-\prod_{i=1}^{d} P\left[\frac{\xi_{i}+\zeta_{i}}{\xi_{i}+\zeta_{i}} \cdot\left(\xi_{i}+\zeta_{i}\right) \frac{\alpha_{i}}{]}\right]
$$

If $\alpha_{i} \geqslant 1$, the corresponding fraction is a polynomial. If $\alpha_{i}=0$, we have $\xi_{i}=x_{a}$, $\zeta_{i}=x_{b}$ for some indexes $a<b$, we use the relation $P\left[\left(x_{a}-x_{b}\right) /\left(x_{a}+x_{b}\right)\right]=1$. Substituting $\left(x_{1}, \ldots, x_{k}\right) \in \Delta_{k}^{n}$, we see that if $\xi_{i}+\zeta_{i}<\alpha_{i}$ for some index $i$, both minuend and the subtrahend take zero value, otherwise $\xi_{i}=\zeta_{i}=0$ for all $i$ with $\alpha_{i}=0$, thus the corresponding factors in the minuend and subtrahend are equal.

For proving (ii), note that we should have $x_{j}=\xi_{s}$ for some $s$, but if $\xi_{s}$ is taken in negative power, then $\zeta_{s}=x_{b_{s}}$ must be taken in positive power and $b_{s}>j$.

Modulo this lemma everything is less or more the same as in previous sections.

Theorem 5.2 The polynomial component of the function $f_{n}\left(x_{1}, \ldots, x_{k}\right)$ equals

$$
\begin{aligned}
P\left[\prod_{1 \leqslant i<j \leqslant k} \frac{x_{i}-x_{j}}{x_{i}+x_{j}} \cdot\left(x_{1}+\cdots+x_{k}\right)^{\frac{n}{}}\right] \\
=\sum_{m_{1}+\cdots+m_{k}=n} \prod_{i<j} \frac{m_{i}-m_{j}}{m_{i}+m_{j}} \cdot \frac{n !}{\prod m_{i} !} \cdot \prod x_{i} \frac{m_{i}}{}
\end{aligned}
$$

(recall that $\left(m_{i}-m_{j}\right) /\left(m_{i}+m_{j}\right)$ for $m_{i}=m_{j}=0$ is equal to 1$)$.

Proof Both parts are polynomials of degree at most $n$, thus it suffices to check that their values at each point $\left(c_{1}, \ldots, c_{k}\right) \in \Delta_{k}^{n}$ are equal. The polynomial component of $f_{n}\left(x_{1}, \ldots, x_{k}\right)$ takes the same values on $\Delta_{k}^{n}$ as the function $f_{n}$. Thus, by Lemma 2.4, it suffices to check that $f_{n}$ vanishes on $\Delta_{k}^{n-1}$. But already the factor $\left(x_{1}+\cdots+x_{k}\right)^{n}$ vanishes on $\Delta_{k}^{n-1}$.

Corollary 5.3 The coefficient $H\left(m_{1}, \ldots, m_{k}\right)$ of the Laurent series

$$
\prod_{1 \leqslant i<j \leqslant k} \frac{x_{i}-x_{j}}{x_{i}+x_{j}} \cdot\left(x_{1}+\cdots+x_{k}\right)^{n}
$$

in monomial $\prod x_{i}^{m_{i}}, m_{i} \geqslant 0$, equals $\prod_{i<j}\left(m_{i}-m_{j}\right) /\left(m_{i}+m_{j}\right) \cdot n ! / \prod m_{i} !$. 
Corollary 5.4 If $u=\prod x_{i}^{m_{i}}$ is a vertex of $S y_{k}, n=\sum m_{i}$, then the number of paths from the origin to $v$ equals

$$
P_{\mathcal{S} y_{k}}(1, u)=\prod_{i<j} \frac{m_{i}-m_{j}}{m_{i}+m_{j}} \cdot \frac{n !}{\prod m_{i} !}
$$

Proof Apply Theorem 1.1 to the function $\varphi=\prod_{1 \leqslant i<j \leqslant k}\left(x_{i}-x_{j}\right) /\left(x_{i}+x_{j}\right)$. Then the result directly follows from Corollary 5.3, so it suffices to check all conditions of Theorem 1.1. Condition (i) follows from Corollary 5.3 for $n=0$ (or just from common sense). In condition (ii) there is nothing to check since 1 is the unique vertex of $\mathcal{S} y_{k}$ with degree 0 . Let us check condition (iii). Assume that $w=\prod x_{i}^{c_{i}}$ is such that $x_{i} w$ is a vertex of $\delta y_{k}$ but $w$ is not. There are two cases: either all coordinates of $w$ are non-negative and $c_{j}=c_{l}>0$ for some $j, l$, or $c_{i}=-1, c_{j}=0$ for all $j \geqslant i$. In the first case apply Corollary 5.3, in the second case apply statement (ii) of Lemma 5.1.

\section{Skew strict Young tableaux}

Here we give an identity corresponding to the formula $[8,17]$ for the number of paths between any two vertices of the graph $\delta y_{k}$, or, in other words, for the number of strict skew Young tableaux of a given shape.

Let $v=\prod_{i=1}^{k} x_{i}^{m_{i}}, m_{1}>m_{2}>\cdots>m_{\ell}>m_{\ell+1}=0=\cdots=m_{k}$, be a vertex of $\delta y_{k}, u=\prod x_{i}^{n_{i}}$ be another vertex of $\delta y_{k}$ and $n_{i} \geqslant m_{i}$ for all $i$ (thus there exists some path from $u$ to $v$ ). Denote $m=\sum m_{i}, n=\sum n_{i}$.

For counting such paths we introduce the following polynomial (Ivanov in [8] attributes it to Okounkov):

$$
\psi_{v}\left(x_{1}, \ldots, x_{k}\right)=\frac{1}{(k-\ell) !} \operatorname{Sym}\left(\prod_{i \leqslant \ell} x_{i} \frac{m_{i}}{i \leqslant \ell, i<j} \prod_{i} \frac{x_{i}+x_{j}}{x_{i}-x_{j}}\right) .
$$

Here Sym $F\left(x_{1}, \ldots, x_{k}\right)=\sum_{\pi} F\left(x_{\pi_{1}}, \ldots, x_{\pi_{k}}\right)$, where summation is taken over all $k$ ! permutations of numbers $1, \ldots, k$. (For concluding that it is indeed a polynomial note that any multiple $x_{i}-x_{j}$ in denominator disappears after natural pairing of summands. It is a super-symmetric polynomial, but we do not use this fact.)

Then define the function

$$
\varphi_{v}\left(x_{1}, \ldots, x_{k}\right)=\prod_{i<j} \frac{x_{i}-x_{j}}{x_{i}+x_{j}} \cdot \psi_{v}\left(x_{1}, \ldots, x_{k}\right) .
$$

The following theorem generalizes results of the previous section.

Theorem 6.1 Denote $g\left(x_{1}, \ldots, x_{k}\right)=\varphi_{v}\left(x_{1}, \ldots, x_{k}\right) \cdot\left(x_{1}+\cdots+x_{k}-m\right) \frac{n-m}{\text {. }}$

(i) The antipolynomial component $Q[g]$ vanishes on the simplex $\Delta_{k}^{n}$. 
(ii) The polynomial component of $g$ has the expansion

$$
P[g]=\sum_{\substack{c_{1}+\cdots+c_{k}=n \\ c_{i} \geqslant 0}} \frac{(n-m) !}{\prod c_{i} !} \cdot \varphi_{v}\left(c_{1}, \ldots, c_{k}\right) \cdot \prod x_{i} \stackrel{c_{i}}{.}
$$

(iii) The number of paths from $v=\prod x_{i}^{m_{i}}$ to $u=\prod x_{i}^{n_{i}}$ equals

$$
\frac{(n-m) !}{\prod n_{i} !} \cdot \varphi_{v}\left(n_{1}, \ldots, n_{k}\right)
$$

Proof (i) Fix a permutation $\pi$ and prove the statement for the antipolynomial component of the corresponding summand in the definition of $\varphi_{v}$. Denote $y_{i}=x_{\pi_{i}}$. Note that up to a sign our summand is

$$
\left(y_{1}+\cdots+y_{k}-m\right) \frac{n-m}{\prod} y_{i} \frac{m_{i}}{\prod_{\ell<i<j}} \frac{y_{i}-y_{j}}{y_{i}+y_{j}} .
$$

We expand $\prod_{\ell<i<j}\left(y_{i}-y_{j}\right) /\left(y_{i}+y_{j}\right)$ as a Pfaffian as explained in the proof of Lemma 5.1 (if $k-\ell$ is odd do the same thing as before: add a vanishing variable; so let $k-\ell=2 d$ be even). Expand this Pfaffian, and take a summand like $\prod_{i=1}^{d}\left(\xi_{i}-\zeta_{i}\right) /\left(\xi_{i}+\zeta_{i}\right)$, where $\left\{\xi_{1}, \zeta_{1}, \ldots, \xi_{d}, \zeta_{d}\right\}=\left\{y_{\ell+1}, \ldots, y_{k}\right\}$.

Expand also $\left(x_{1}+\cdots+x_{k}-m\right) \frac{n-m}{n}$ by the Chu-Vandermonde identity (2) as a linear combination of terms like

$$
\left(y_{1}-m_{1}\right) \underline{\alpha_{1}} \cdots\left(y_{\ell}-m_{\ell}\right) \frac{\alpha_{\ell}}{}\left(\xi_{1}+\zeta_{1}\right) \underline{\beta_{1}} \cdots\left(\xi_{d}+\zeta_{d}\right) \underline{\beta_{d}}
$$

$\sum \alpha_{i}+\sum \beta_{i}=n-m$. Thus it suffices to prove that the antipolynomial component of the following product vanishes on $\Delta_{k}^{n}$ :

$$
\prod_{i \leqslant \ell} y_{i} \frac{m_{i}+\alpha_{i}}{\prod_{i=1}^{d}} \frac{\xi_{i}-\zeta_{i}}{\xi_{i}+\zeta_{i}} \cdot\left(\xi_{i}+\zeta_{i}\right) \frac{\beta_{i}}{.}
$$

The variables are separated, hence the polynomial component $P[\Pi \cdots]$ of the product is a product $\prod P[\cdots]$ of polynomial components. It suffices to verify that whenever $y_{i}, \xi_{i}, \zeta_{i}$ are non-negative integers with sum at most $n$, the values of $\prod(\cdots)$ and $\prod P[\cdots]$ are equal. If $\beta_{i} \geqslant 1$, the corresponding fraction $\left(\xi_{i}-\zeta_{i}\right) /\left(\xi_{i}+\zeta_{i}\right) \cdot\left(\xi_{i}+\zeta_{i}\right) \frac{\beta_{i}}{y_{i}}$ is a polynomial. If $\beta_{i}=0$, we have $\xi_{i}=x_{a}, \zeta_{i}=x_{b}$ for some indexes $a<b$, then $P\left[\left(x_{a}-x_{b}\right) /\left(x_{a}+x_{b}\right)\right]=1$. Substituting $\left(x_{1}, \ldots, x_{k}\right) \in \Delta_{k}^{n}$, we see that if $y_{i}<m_{i}+\alpha_{i}$ or $\xi_{i}+\zeta_{i}<\beta_{i}$ for some index $i$, then both products take zero value, otherwise $\xi_{i}=\zeta_{i}=0$ for all $i$ with $\beta_{i}=0$, thus the corresponding fractions (values of the function and its polynomial part) are equal.

Note that as in Lemma 5.1 we may also conclude that if $c_{1}, \ldots, c_{k}$ are integers such that $c_{j}<0, c_{i} \geqslant 0$ for $i=j+1, \ldots, k$, then $\left[\prod x_{i}^{c_{i}}\right] g=0$. 
(ii) The values of $P[g]$ on $\Delta_{k}^{n}$ are the same as values of $g$. Any summand of the above expansion for $g$ vanishes on $\Delta_{k}^{n-1}$. Thus it suffices to use Lemma 2.4.

(iii) Apply Theorem 1.1 to the function $\varphi_{v}$ (or its leasing part, it is a matter of taste). It suffices to check all conditions of Theorem 1.1. Conditions (i) and (ii) follow from the above expansion of $g$ (with $n=0$ ). There are exactly $(k-\ell)$ ! permutations with $y_{i}=x_{i}, i=1, \ldots, \ell$, for each of them we get coefficient 1 in the monomial $v$ and coefficient 0 in other monomials of degree $m$. For other permutations we do not get non-zero coefficients in monomials which are vertices of $\delta y_{k}$. Let us check condition (iii). Assume that $w=\prod x_{i}^{c_{i}}$ is such that $x_{i} w$ is a vertex of $\mathcal{S} y_{k}$ but $w$ is not. There are two cases: either all coordinates of $w$ are non-negative and $c_{j}=c_{l}>0$ for some $j, l$, or $c_{i}=-1, c_{j}=0$ for all $j \geqslant i$. In the first case apply identity (8), in the second case apply the above remark after the proof of part (i) of the theorem.

\section{Concluding remarks}

For the sake of convenience we summarize here the list of functions $\varphi$ for which coefficients of $\varphi\left(\sum x_{i}\right)^{N}$ count the number of paths from the vertex $v$ in some graded graph $G$.

(a) $\varphi=\prod x_{i}^{m_{i}}, v=\prod x_{i}^{m_{i}}, G$ is the multidimensional Pascal graph $\mathcal{P}_{k}$.

(b) $\varphi=\operatorname{det}\left(x_{i}^{m_{i}}\right), m_{1}<m_{2}<\cdots<m_{k}, v=\prod x_{i}^{m_{i}}, G$ is the Young graph $y_{k}$ formed by strictly increasing sequences.

(c) $\varphi=\prod_{i<j}\left(x_{i}-x_{j}\right) /\left(x_{i}+x_{j}\right), v$ is the origin, $G=\mathcal{S} Y_{k}$ is the graph of strict Young diagrams. When $v$ is not the origin, the corresponding function $\varphi_{v}$ should be multiplied by the Okounkov polynomial, as described in Sect. 6 .

We may observe that functions $\varphi$ in examples (b) and (c) vanish on the "boundary" of the corresponding graph considered as a subgraph of $\mathcal{P}_{k}$. However, we do not know how to guess the function of example (c) without knowing it a priori. Hopefully, the answer to this question may help to generalize this machinery to other graphs.

Another question which has to be answered is how identities with falling factorials are related with asymptotics of dimensions.

Acknowledgments This work originated from collaboration with Gyula Károlyi, Zoltán Nagy and Vladislav Volkov. The idea to study the graph of strict diagrams in the same spirit belongs to Anatoly Vershik. To all of them the author is really grateful. He is also grateful to the referees for useful suggestions and especially for pointing out several important papers on the subject.

\section{References}

1. Alon, N.: Combinatorial Nullstellensatz. Combin. Probab. Comput. 8(1-2), 7-29 (1999)

2. Alon, N., Nathanson, M.B., Ruzsa, I.: The polynomial method and restricted sums of congruence classes. J. Number Theory 56(2), 404-417 (1996)

3. Balandraud, É.: An addition theorem and maximal zero-sum free sets in $\mathbb{Z} / p \mathbb{Z}$. Israel J. Math. 188, 405-429 (2012)

4. Balandraud, É.: Erratum to: "An addition theorem and maximal zero-sum free sets in $\mathbb{Z} / p \mathbb{Z}$ ". Israel J. Math. 192(2), 1009-1010 (2012) 
5. Borodin, A., Olshanski, G.: Harmonic functions on multiplicative graphs and interpolation polynomials. Electron. J. Combin. 7, R28 (2000)

6. Frame, J.S., Robinson, G. de B., Thrall, R.M.: The hook graphs ofthe symmetric groups. Canad. J. Math. 6, 316-324 (1954)

7. Fulmek, M.: Viewing determinants as nonintersecting lattice paths yields classical determinantal identities bijectively. Electron. J. Combin. 19(3), P21 (2012)

8. Ivanov, V.N.: Dimensions of skew-shifted young diagrams and projective characters of the infinite symmetric group. J. Math. Sci. (N. Y.) 96(5), 3517-3530 (1999)

9. Karasev, R.N., Petrov, F.V.: Partitions of nonzero elements of a finite field into pairs. Israel J. Math. 192(1), 143-156 (2012)

10. Károlyi, G., Nagy, Z.L.: A simple proof of the Zeilberger-Bressoud $q$-Dyson theorem. Proc. Amer. Math. Soc. 142(9), 3007-3011 (2014)

11. Károlyi, G., Nagy, Z.L., Petrov, F.V., Volkov, V.: A new approach to constant term identities and Selberg-type integrals. Adv. Math. 277, 252-282 (2015)

12. Knuth, D.E.: Two notes on notation (1992). arxiv:math/9205211

13. Lasoń, M.: A generalization of combinatorial Nullstellensatz. Electron. J. Combin. 17(1), N32 (2010)

14. Okounkov, A.: On Newton interpolation of symmetric functions: a characterization of interpolation Macdonald polynomials. Adv. in Appl. Math. 20(4), 395-428 (1998)

15. Okounkov, A., Olshanski, G.: Shifted Schur functions. St. Petersburg Math. J. 9(2), $73-146$ (1997)

16. Olshanski, G., Regev, A., Vershik, A.: Frobenius-Schur Functions. In: Joseph, A., Melnikov, A., Rentschler, R. (eds.) Studies in Memory of Issai Schur. Progress in Mathematics, vol. 210, pp. 251299. Birkhäuser, Boston (2003)

17. Thrall, R.M.: A combinatorial problem. Michigan Math. J. 1(1), 81-88 (1952) 